\title{
Le Néolithique et l'Age du Bronze en France: regards sur une recherche et un patrimoine
}

\author{
J. GuILAINE *
}

Les pages qui suivent n'ont pas la prétention d'évoquer tous les aspects concernant l'exploitation scientifique, la protection ou la mise en valeur du patrimoine français en matière de Néolithique et d'Age du Bronze. On a seulement mis l'accent sur quelques questions d'actualité.

\section{UN PEU D'HISTOIRE: UNE DISCIPLINE JEUNE}

L'étude du Néolithique n'a réellement débuté en France sur des bases solides qu'à partir des années 50 . C'est donc une science plutôt jeune. L'état de la recherche ou la prise en considération du patrimoine sont donc un peu le reflet de cette relative jeunesse. Avant 1950 on n'avait guère surmonté les handicaps stratigraphiques et le Néolithique, période d'environ trois millénaires, était souvent conçu comme une entité, comme un tout sans nuance chronologique ou spatiale. L'Age du Bronze (un millénaire et demi) bénéficiait par contre d'un intérêt plus soutenu, dû essentiellement à une certaine tradition d'études typologiques: les instruments de métal provenant des nombreux dépôts du territoire français étaient classés selon leur morphologie et calés en chronologie relative. Mais ici aussi les objets restaient la source d'information principale: les

\footnotetext{
* CNRS/EHESS/Sous-Direction de l'Archéologie.
} 
habitats n'étaient que peu étudiés, les sépultures guère plus. En France la recherche préhistorique était essentiellement vouée à l'étude des temps paléolithiques, périodes nobles dominées par l'approche de la typologie lithique et, après sa reconnaissance, par l'étude de l'art des cavernes. A l'autre pôle de la chaîne chronologique, l'intérêt pour la période gauloise (2e Age du Fer), largement encouragée par certains gouvernants (cf. Napoleón III) ne laissait pas indifférent: on considérait en effet cette période comme la première expression de l'unité nationale et de la résistance a l'envahisseur, notions qui, on le sait, on pesé lourd dans les Manuels d'Histoire de naguère. Entre ces deux extrêmes, le Néolithique et l'Age du Bronze ont souvent été considérés comme les derniers temps de la Préhistoire, des sortes d'épiphénomènes du Paléolithique. La tradition de la taille de la pierre, dans un pays où l'étude de la typologie tenait lieu de guide méthodologique principal, sous-tendait ce lien conceptuel. L'originalité des temps néolithiques et protohistoriques -c'est-à-dire la mise en place du monde agricole- était donc en fait sous-évaluée dans la mesure où ces périodes concernent essentiellement des populations totalement différentes, dans leur système socio-économique de celles des chasseurs-cueilleurs paléolithiques. On n'insistera jamais assez sur ce point: le succés des études paléolithiques a été un frein à une analyse poussée de l'époque néolithique. Heureusement une réaction bénéfique s'est produite et les études sur la Préhistoire récente sont désormais en constant développement. Effet de balancier? Aujourd'hui c'est la recherche de terrain paléolithique qui, en France, cherche un second souffle. Par ailleurs le milieu des archéologues trop longtemps focalisé sur les problèmes typologiques, en venait à oublier l'essentiel de ces périodes néolithiques et protohistoriques: la transformation radicale du paysage sous l'effet de la pression des premières communautés agricoles. Il a fallu que des géographes ou des historiens (G. Roupnel, $M$. Bloch et récemment encore $F$. Braudel) montrent tout l'intérêt qu'il y avait à éclaircir les questions liées à cette première humanisation du sol français.

Enfin pendant de longues années le poids du mythe oriental dans la mise en place et l'évolution des populations de la Préhistoire récente a contribué à situer les civilisations europeennes dans un état de dépendance culturelle (et chronologique) par rapport aux civilisations est-méditerranéennes. On sait comment les datations absolues (grâce au C 14 notamment) ont contribué à libérer les néolithiciens occidentaux du carcan de l'interdépendance des cultures, soulignant par là même la vigueur et l'originalité de certains traits culturels européens. 


\section{TROIS A QUATRE ETAPES DANS L'APPROCHE SCIENTIFIQUE}

On peut, systématiquement, considérer que l'étude du Néolithique et de l'Age du Bronze en France -et donc celle des sites de ces périodes- s'est déroulée depuis quelques décennies selon trois à quatre stades dont les derniers au moins sont en grande partie concomitants et dont aucun ne peut être considéré aujourd'hui comme clos.

- D'abord a été mise en évidence l'identification des principaux faciès régionaux et leur articulation chronologique. Cette phase débutée dans les années 50 (et peut-être avant) est passée, pour le Néolithique en particulier, par le repérage et la fouille de site stratifiés en grotte ou sous abri, essentiellement répartis dans les régions karstiques du Sud et du Sud-Est de la France: abri de Châteauneuf-les-Martigues (Bouchesdu-Rhône), grotte de la Madeleine (Villeneuve-les-Maguelonne, Hérault), grotte-doline de Roucadour (Thémines, Lot), grotte Gazel et abri de FontJuvénal (Aude), Baume Fontbrégoua (Var), abri d'Araguina-Sennola (Corse), etc. D'autres exemples existent pour l'Age du Bronze (cf. Marsa, Beauregard) (Lot), parfois en plein air (cf. Videlles, Seine-et-Marne). Grottes et abris ont l'avantage d'avoir constitué des lieux de fréquentation pendant plusieurs millénaires et les vestiges stratifiés qu'ils recèlent ont permis d'asseoir la charpente chronologique et régionale des civilisations étagées du $5 \mathrm{e}$ au 1er millénaire b.c. Dans les régions où de telles stratigraphies n'existaient pas la définition des faciès s'est réalisée à partir de mobiliers sépulcraux (cf. les faciès mégalithiques de l'Ouest de la France, les séries issues des nécropoles du Bronze final) ou le contenu de dépôts (Age du Bronze armoricain, du bassin parisien ou de l'Est).

- Par la suite on assista progressivement à la mise en place de recherches extensives sur les sites de plein air pour décaper les restes des villages du Néolithique et de la Protohistoire. En ce domaine la France faisait preuve jusqu à peu de temps d'un retard tout à fait inquiétant comparativement à plusieurs pays de l'Europe centrale ou de l'Europe du Nord-Ouest. Le detonateur est venu, dans le courant des années 1970, de l'opération lancée par B. Sousky dans la vallée de l'Aisne sur le site néolithique danubien de Cuiry-les-Chaudardes, opération encore en cours aujourd'hui. Depuis plusieurs années le Conseil Supérieur de la Recherche Archéologique, à travers son programme P27, aide à la réalisation de fouilles extensives sur des habitats du Néolithique et de l'Age du Bronze. Plusieurs variétés de sites ont bénéficié ou bénéficient de cette politique tout à fait d'actualité. Ainsi parmi les divers exemples peut on citer les premiers villages danubiens (Cuiry, déjà mentionné; Larzi- 
court, Haute-Marne), les agglomérations chasséennes du Sud (Villeneuve-Tolosane, Haute-Garonne; St Paul-Trois-Châteaux, Drôme), les habitats «mégalithiques» armoricains (Loc Mariaquer, Morbihan), les sites lacustres (Clairvaux, Jura; Charavines, Isère), les sites chasséens et Michelsberg du bassin parisien et de ses marges (Noyen, Seine-et-Marne; Mairy, Ardennes), les camps à fossés de l'Ouest (Nieul-sur-l'Aurise, Vendée; Machecoul, Morbihan), les sites néolithiques et chalcolithiques du Languedoc (Roquemengarde et Cambous, Hérault). La fouille en extensif d'habitats de l'Age du Bronze a connu un décollage plus tardif mais désormais réel: Le Fort Harrouard (Eure-et-Loir), site déjà largement occupé au Néolithique et au Chalcolithique; Dampierre-sur-le-Doubs (Jura), etc.

- Autre étape de la recherche: l'approche du paléoenvironnement et du paysage peu à peu modifiés par les premières sociétés agricoles et métallurgiques. Cette recherche qui met en jeu toutes les disciplines de l'écologie préhistorique et historique (sédimentologie, palynologie, anthracologie, dendrochronologie, carpologie, malacologie, zoologie, etc.) s'est progressivement mise en place à partir des années 1960 mais en s'intensifiant au cours de ces deux dernieres décennies. Sa qualité et ses résultats reposent beaucoup sur divers chercheurs ou équipes du CNRS. Trois laboratoires s'investissent plus particulièrement dans cette recherche paléoécologique: le Centre de Recherches Archéologiques de Valbonne (CNRS), le Centre National de Préhistoire à Périgueux (Ministère de la Culture), le Laboratoire de Chrono-Ecologie à Besançon (CNRS/ Culture). L'essentiel de cette recherche repose souvent sur de jeunes chercheurs travaillant sous contrat, fréquemment de façon précaire. L'émergence récente de laboratoires privés (cf. Archéolab à St Milaire du Rogier, Isère) remplissant des fonctions de service est à noter. Les recherches de pétrographie sur les outillages lithiques ainsi que les analyses métalliques sur les cuivres et les bronzes protohistoriques (celles-ci conduites pour l'essentiel au laboratoire d'Anthropologie de l'Université de Rennes 1) peuvent être considérées comme partie intégrante de cette recherche dans la mesure oú les mutations de l'environnement sont liées aux incidences des contraintes économiques. De même l'analyse des finages s'inscrit-elle dans la même démarche.

- Pour ne rien laisser dans l'ombre, il faudrait aussi évoquer divers axes de recherche en archéologie "sociale", tous d'actualité. Citons par exemple les recherches anthropologiques orientées vers les rites funéraires, la paléodémographie, la paléopathologie. Ou encore l'étude, en Ar- 
morique, des relations chronologiques entre les grands menhirs décorés, véritables stèles, dressés puis brisés avant d'être réutilisés dans la construction des tombes mégalithiques (dolmens du Golfe du Morbihan). On peut aussi évoquer les recherches d'ethnoarchéologie destinées à nous éclairer sur le comportement des sociétés du Néolithique ou de l'Age du Bronze. L'optique peut être purement technique: taille ou polissage des matériaux lithiques et osseux, confection de la terre cuite, fonte d'objets en métal. Mais parfois, derrière une recherche de caractère technique --par exemple l'expérience de la construction d'un mégalithe réalise à Bougon, Deux-Sèvres - se profilent des enseignements sur l'économie, la démographie, la psychologie, la hiérarchie sociale, la religion. Les expériences actuelles d'agriculture expérimentale (Chassemy, Aisne; Jalès, Ardèche) sont aussi très éclairantes.

\section{UN PATRIMOINE DIFFICILE A ETUDIER, A GERER, A METTRE EN VALEUR}

\section{a) Des monuments en matériaux périssables}

On peut, au delà des objets (outils du quotidien, armes, parures, céramiques, etc.) dont la conservation trouve naturellement place dans les Musées et les nombreux Dépôts de Fouilles, considérer qu'il existe, très schématiquement, deux types de monuments pour les périodes ici concernées:

- Des bâtiments construits en "dur» (pierre) ou à charpente dans laquelle la pierre joue un rôle important et donc plus facilement conservés et conservables. Exemple: les tombes mégalithiques, les menhirs, certains tumulus à chambre de pierre ou à volume important, des habitats construits en pierre (cf. sites Fontbouisse du Midi ou sites torréens de Corse)

- des bâtiments ou des structures autrefois bâtis en bois, terre et torchis et, de ce fait, très vulnérables: cabanes, palissades, systèmes de protection, fours, etc. 
Si les premiers ont souvent été protégés par la solidité de leur matériau, les seconds ont été précocement ruinés et, par la suite, carrément détruits plus ou moins totalement par les pratiques culturales. On mesure d'emblée les difficultés d'approche au plan scientifique.

- Beaucoup de sols d'habitat n'existent plus. On ne dispose souvent que d'éléments tronqués, plus ou moins interprétables, en général les parties les plus profondes de structures en creux: les fonds de fossés, les fonds de silos, les parties inférieures des fosses, la partie la plus enterrée des poteaux, etc. On connaît ainsi d'assez nombreux plans de cabanes danubiennes mais aucun sol d'habitat. Les grands sites à enceintes (Centre-Ouest) n'ont conservé que leurs fossés, rarement les sols des aires habitées.

- Les éléments découverts tronqués rendent l'interprétation malaisée. Les longues aires rectangulaires "en fosse", emplies de galets brûlés, du Chasséen toulousain ont été interprétées tantôt comme des assises de cabanes tantôt comme de grandes structures de combustion.

La plupart du temps donc un site n'est repérable (au sol ou par voie aérienne) que par les taches de terre sombre qui en subsistent, restes de structures en creux partiellement épargnées. L'étude se concentrera donc sur une fouille qui est en fait une lecture de structures tronquées à partir desquelles il faudra reconstituer intellectuellement les parties aériennes ou enterrés disparues. Certes cette situation est assez générale mais la France, vieux pays agricole à dominante rurale, a subi plusieurs millénaires de mise en culture qui ont très largement mutilé beaucoup de ses sites néolithiques et protohistoriques.

b) Architecture de bois et de terre: comment la mettre en valeur?

L'absence fréquente (mégalithes et tumuli exceptés) de monuments spectaculaires dans le Néolithique et l'Age du Bronze tient donc, on l'a $\mathrm{vu}$, à la nature même des matériaux de construction - bois et terre - qui ont été les premières causes de la non-conservation des bâtiments. De ceux-ci il ne subsiste rien d'aérien, rien de visible. Le seul moyen de valoriser une telle architecture disparue réside donc dans des reconstruc- 
tions, grandeur nature, de maisons, d'enclos ou de dispositifs de protection. Se limiter en effet à l'observation d'empreintes négatives au sol présente un aspect par trop réducteur. Certes ces restitutions ne vont pas sans poser des problèmes d'élévation des murs, de disposition des charpentes qu'il faut tâcher de résoudre selon des régles déontologiques strictes: il n'est pas question d'inventer. L'intérêt de ces restitutions est réel. Qu'on se souvienne du succés d'audience de films (TF1) de J. Audoir et R. Clarke ("L'argile et le grain", "Nos ancêtres les gaulois"), télédiffusés à plusieurs reprises et dans lesquels on assiste à la reconstitution d'une maison néolithique ou d'une ferme gauloise. Et comment ne pas souligner ici le succès populaire de "l'archéodrome" de Beaune ou, près d'une jonction autoroutière essentielle du réseau français, une aire de repos présente au voyageur une restitution grandeur nature de plusieurs variétés de bâtiments de la protohistoire française, depuis les habitations danubiennes jusqu'aux circonvallations d'Alésia. Les reconstitutions sont bel et bien la seule façon de mieux faire connaître l'architecture néolithique et protohistorique. D'autres projets sont à l'étude: ainsi la reconstitution d'une petite localité néolithique à Chalain (Jura).

\section{UN PROBLEME PREOCCUPANT: LE MEGALITHISME}

Avec quelque 6.000 dolmens, 2.000 menhirs auxquels viennent s'ajouter les alignements (dont quelque 4.000 pierres levées pour la seule région de Carnac), la France possède sans doute le plus grand conservatoire mégalithique du monde. L'effectif même rend difficile une politique de conservation qui prendrait en compte la totalité des monuments existants. C'est pourtant vers une attitude maximaliste qu'il faudrait aller: classement ou inscription par des COREPHAE dans chaque département de la totalité des monuments recensés, protection, restauration, entretien obligatoire, selon le statut, par les administrations ou les propriétaires responsables (Monuments Historiques, Conseils Généraux, Communes, particuliers) qui pourraient faire appel aux sociétés de protection de la nature ou aux associations mandatées par la Direction des Antiquités. II conviendrait d'abord de reprendre, département par département, le vieil inventaire de De Mortillet, en partie caduc, et le remplacer par un inventaire "léger» mais efficace. L'idéal reste, bien entendu, l'inventaire "lourd" c'est-à-dire la publication détaillée par département de l'Inventaire des Mégalithes de la France dans les Suppléments a la revue "Gallia- 
Préhistoire» (CNRS): une quinzaine de départements ont seulement été terminés et édités à ce jour.

Inventaires et classements ne constitueront pourtant qu'une étape. Si l'on veut valoriser et conserver les monuments mégalithiques de la France, il faut une politique volontariste qui associe toutes les administrations qui ont en charge le milieu natural. Les mégalithes sont fragiles: leur conservation est l'affaire de tous. Or souvent, dans des régions dépeuplées, les seules personnes qui s'y intéressent sont malheureusement les fouilleurs clandestins; par leurs recherches inconsidérées des mobiliers, ils déchaussent les piliers et provoquent à terme la ruine des monuments. II est aussi vrai que, dans l'ensemble, le patrimoine mégalithique survit sans aucun entretien dans un état de désintéressement attristant. On sait trop également que certains reboisements effectués par telle administration ont abouti à la destruction de monuments mégalithiques.

II importe surtout d'effectuer des restaurations de grande envergure pour les ensembles monumentaux les plus remarquables. Les exemples de Barnenez (Côtes-du-Nord) et de Bougon (Deux-Sèvres) doivent être suivis. Dans le cadre d'une loi-programme visant à restaurer et mettre en valeur les principaux sites archéologiques de France (une trentaine), un premier train de mesures va s'attacher à restaurer très profondément les alignements de Carnac et les grandes chambres mégalithiques de cette région: Table des Marchands, Mané-Lud; Mané-er-Hroek, les Pierres Plates, le Petit-Mont, Gavrinis. Dégagement des monuments, mise en évidence des paraments du tumulus, construction des murets si possible jusqu'à leur hauteur primitive, révision des architectures, présentation intérieure sont prévus. Le cas de Gavrinis où les gravures s'érodent rapidement fera peut-être l'objet, sur l'îlot même, d'une reconstitution grandeur nature: un projet dans ce sens a été récemment proposé. Parmi les sites qui feron l'objet d'une aide dans le cadre de la loi-programme on retrouve aussi les hypogées de Fontvieille près d'Arles, ensemble architectural unique sur le territoire français.

\section{LE RENOUVEAU DE L'ARCHEOLOGIE LACUSTRE}

Depuis quelques années la France a pris conscience que son capital en archéologie lacustre méritait une meilleure exploitation scientifique. Etablis en bordure des lacs de l'Est français, aujourd'hui ennoyés ou situés "à terre" à proximité de la ligne de rivage, des habitats du Néoli- 
thique ou de l'Age du Bronze ont été depuis longtemps repérés. Leur richesse en vestiges mais aussi l'excellente conservation des infrastructures de bois ainsi que des instruments ligneux de la vie quotidienne en ont fait malheureusement des sites-martyrs, pillés par les collectionneurs. Des recherches archéologiques appuyées d'études poussées sur le paléoenvironnement et le calage dendro-chronologique ont abouti ces dernières années à des résultats d'une rare qualité et d'une précision chronologique inégalée. Les exemples de Charavines (Isère) et de Clairvaux (Jura) sont à mentionner. II convient toutefois de rester extrêmement vigilants car ces sites constituent finalement un capital limité, d'intérêt unique mais largement menacé. On en a eu encore tout récemment la démonstration avec les stations du lac de Chalain (Jura). Le maintien de bas niveaux de plans d'eau ( 2 à $3 \mathrm{~m}$ sous la cote d'origine) a entraîné le glissement de paquets entiers de couches archéologiques dans le lac; ainsi ont disparu de nombreux objets de bois et de restes végétaux en même temps que de grandes parties de couches en place. La mise à sac généralisée de secteurs entiers par les collectionneurs, le surpeuplement en été avec plusieurs centaines de baigneurs chaque jour, les aménagements de plages ou de parkings feront peu à peu disparaître si l'on ne réagit pas, les derniers lambeaux des "cités lacustres". Un plan de sauvetage programmé de grande ampleur a donc été mis en place à Chalain sur une occupation du Néolithique final. A Chens-sur-Léman (Haute-Savoie) c'est sur un village ennoyé de l'Age du Bronze que va incessamment débuter une recherche de grande ampleur.

\section{QUELQUES POINTS FAIBLES}

Parmi les points faibles de la recherche française en Néolithique et Ages des Métaux, points qui se doublent d'un certain désintérêt en matière de patrimoine, j'évoquerai la question de l'art rupestre, des structures agraires et des tumuli protohistoriques.

- L'art rupestre protohistorique: Seul le vaste ensemble du MontBego (Tende, Alpes-Maritimes) fait depuis plusieurs années l'objet d'un inventaire et d'une recherche de longue haleine. Le site sera protégé et mis en valeur dans le cadre de la toute prochaine loi-programme. Toutefois la France a rarement su s'intéresser à son art rupestre post-glaciaire. Autant les grottes ornées paléolithiques sont l'objet d'un légitime mouve- 
ment de conservation, autant l'art néolithique, protohistorique voire historique (car en ce domaine les questions de datation restent très embrouillées) reste négligé, abandonné dans une sorte de semi-amateurisme. Une situation à redresser.

- Les structures agraires. C'est ici un sujet passionnant et d'intérêt capital pour mieux approcher le découpage parcellaire et les techniques des sociétés rurales de la préhistoire récente. En ce domaine la recherche britannique reste évidemment un modèle. Les resultats qu'elle a su obtenir dans la mise en évidence des Celtic Fields constituent une donnée capitale en matière d'histoire agraire. En France tout est à peu près à faire. Les structures fossiles ne manquent pourtant pas, notamment en moyenne montagne où leur conservation a été plus aisée par suite d'une anthropisation parfois moins active. II reste que les archéologues français, rebutés au départ par les problèmes de datation chronologique, sont restés en dehors du débat. Seuls les parcellaires antiques ou médiévaux ont intéressé les spécialistes. Un thème de recherche donc à fonder, en commençant par intensifier l'archéologie "hors-site». Une excellente opération est toutefois à citer: la mise en évidence à Pagny-sur-Moselle (Meurtheet-Moselle) de vastes surfaces de défrichements avec aires de brûlis et épandage des cendres pour amander les terres. Ces pratiques sont datables ici du Bronze final II.

- Les tumulus protohistoriques. En France les sépultures sous tertre deviennent fréquentes dès le Bronze ancien-moyen pour devenir le type de tombe le plus courant au 1er Age du Fer. Détruits pour la plupart depuis longtemps dans les régions de plaine ou de collines par les pratiques culturales, beaucoup de tumulus avaient subsisté jusqu'au $19 \mathrm{e}$ siècle en milieu de moyenne montagne. C'est par milliers que ces tombes parsemaient certains secteurs du Jura, des Alpes, du Massif Central ou des Pyrénées. Un engouement incontrôlé pour la fouille de ces tertres, au siècle dernier, a constitué le premier avatar sérieux: des centaines de ces tumulus ont été éventrés, dans le but d'en découvrir les mobiliers. Le bilan de ces premières recherches est souvent très mince. Aujourd'hui, la conquête de nouveaux espaces cultivables en moyenne montagne entraîne des remembrements, des modifications de parcellaires et surtout une reprise des labours ou de travaux qui se soldent par la destruction accélérée de nombreux tertres alors que souvent n'existe aucune carte archéologique dans ces régions d'altitude (cf. le plateau de Lannemezan, 
Hautes-Pyrénées, dans les années 1950-1960). Qui dira le capital à jamais perdu par la France dans ce domaine?

\section{ARCHÉOLOGIE PREVENTIVE ET CARTE ARCHÉOLOGIQUE}

La première des nécessités pour gérer et protéger un patrimoine consiste d'abord à le bien connaitre. Un inventaire précis des archives du sol c'est-a-dire des sites enfouis constitue donc le point de départ obligé de toute politique. Or l'archéologie de prospection, aux fins de constitution d'une carte archéologique fiable est, en France, une notion récente. Trop longtemps imprégnée d'amateurisme, la prospection n'a longtemps été conçue que comme un écrêmage de sites bien connus ou la découverte de gisements jalousement conservés secrets par quelques prospecteurs impénitents ou signalés avec une imprécision délibérée. Grâce aux efforts du Conseil Supérieur de la Recherche Archéologique et de la Sous-Direction de l'Archéologie les prospections-inventaires (aériennes, au sol, lacustres, fluviales, en milieu marin), sont devenu une composante essentielle de la recherche de terrain. II faut s'en réjouir. Le but projeté est la constitution à la fois d'une cartographie et d'une banque de données consultables dès qu'une menace (travaux urbains ou ruraux, agrandissement du réseau routier, etc.) peut se manifester.

A un problème d'inventaire doit immanquablement se juxtaposer une politique de protection et d'achat. Ceci vaut particulièrement pour les sites néolithiques ou protohistoriques qui ont peu attiré l'attention des pouvoirs publics comparativament aux grottes ornées paléolithiques ou aux grands bâtiments romains. Le risque est particulièrement grand, pour les habitats néolithiques de plaine devenus très vulnérables en raison des techniques de la mécanisation, de l'extension du bâti, des grands travaux routiers, des propriétaires désireux de remodeler leur exploitation. Une mesure de classement a réussi par exemple à sauver l'un des derniers grands sites chasséens subsistant en Midi-Pyrénées. Mais le classement est insuffisant. II convient de constituer dans les zones fortement anthropisées de véritables réserves scientifiques pour les futures générations de chercheurs. Sur les terrains pauvres ou en friche (cf. garrigues du Languedoc ou domaine caussenard) où le sol est peu cher et le nombre de sites néolithiques et protohistoriques élevé (dolmens, tumulus, etc.), l'achat de zones sensibles est également souhaitable, politique à associer autant que faire se peut avec celle des parcs régionaux. 


\section{QUELLE POLITIQUE POUR DEMAIN?}

Science récente, l'étude du Néolithique et de l'Age du Bronze ne cesse en France de développer. Dans le memê temps scientifiques et administratifs découvrent chaque jour la partialité du patrimoine parvenu jusquà nous, sa fragilité, les menaces qui pèsent sur lui. La mécanisation reste la cause essentielle de l'hémorragie permanente contre laquelle luttent de leur mieux les Directions d'Antiquites, les chercheurs du CNRS, les rares universitaires impliqués dans l'étude de ces périodes. II va de soi que seule l'union de toutes les forces vives intervenant dans l'Archéologie nationale (SDA, CNRS, Education Nationale) peut avoir quelque efficacité à la fois vis a vis des pouvoirs publics, des élus, des aménageurs ou de l'opinion. Toute division est nuisible. De grands pôles régionaux regroupant Directions d'Antiquités, équipe(s) du CNRS, institutions de l'enseignement supérieur voire musées devraient dans un proche avenir constituer à la fois des centres de formation théorique et pratique, des lieux d'obtention de diplomes ou de thèses, des centres de recherche, des officines où s'elaboreraient les politiques régionales ou interrégionales, des centres de documentation, de protection et de valorisation du patrimoine. Utopie? Peut-être tant que les considérations personnelles l'emporteront sur l'intérêt général. Mais évolution inévitable.

Aujourd'hui l'entrée en force des aménageurs dans l'investissement de l'archéologie de sauvetage en France a permis l'ouverture de nombreux chantiers largament subventionnés avec emploi de personnel rétribué sous contrat. Malheureusement pour les périodes qui nous concernent ici, cette archéologie de sauvetage intéresse sortout les périodes historiques et, fréquemment, le domaine urbain. Le Néolithique et l'Age du Bronce sont rarement concernés par ces opérations '. La recherche sur ces périodes s'effectue donc le plus souvent dans le cadre de Fouilles Programmées ou de Sauvetages Programmés, conduits sur crédits d'Etat, aux moyens donc limités. De sorte qu'une espèce de contradiction existe en France entre d'une part une archéologie néolithique et protohistorique dynamique qui a surmonté ses complexes d'infériorité et, d'autre part, les moyens dont elle dispose. Viennne le jour où grands chantiers de fouilles programmées - type Mont-Beuvray- s'ouvriront plus

- Citons tout de même la belle opération de Loc Mariaquer (Morbinan) financée sur crédits de sauvetage 66-20, la série des fouilles de sauvetage effectuées depuis plusieurs annés dans la vallée de l'Aisne ou le chantier, en cours d'ouverture, de Chens-sur-Léman (Haute-Savoie). 
largement sur des sites abritant les vestiges de paléocommunautés agricoles. Notre pays pourra alors élaborer de grands desseins et réaliser des opérations de réelle envergure telles qu'on en connaît dans quelques pays européens. 
